\title{
Selected Topics on Business Informatics: Editorial Introduction to Issue 20 of CSIMQ
}

\author{
Jelena Zdravkovic* \\ Stockholm University, Box 7003, Kista, 16407, Sweden \\ jelenaz@dsv.su.se
}

Business Informatics is the scientific discipline targeting business processes and related phenomena in their socio-economical context, including companies, organizations, administrations, and society in general. As a field of study, it endeavors to take a systematic and analytic approach in adopting a multi-disciplinary orientation that draws theories and practices from the fields of management science, organizational science, computer science, systems engineering, information systems, information management, social science, and economics information science.

This issue complements the previous issue in the way that it aggregates some prominent research results of the 17th International Conference on Perspectives in Business Informatics Research (BIR2018) in Stockholm, Sweden, in September 24-26, 2018 together with a recent research in the Business Informatics discipline and thus makes this issue highly actual. The topics of the issue concern improvements of enterprise architecture modeling and management in the directions of automation, adaptivness, digitalization and security with privacy.

The thematic issue collection opens with the article entitled "Situation Analytics and Modelbased User Interface Development: A Synergetic Approach for Building Runtime-Adaptive Business Applications", which proposes an architecture that combines a situation analytics platform with model-based user interface construction tools in order to support development of adaptive business applications with enhanced user experience and task-accomplishment characteristics. The main focus is set on the software components and tools for observing and tracking the user, data types for modeling and recognizing situations, as well as to modeling structural actions for generating dynamic adaptations. The situation recognition capabilities and adaptive functionality of the system are demonstrated for online portals for long distance travel booking.

The second article, "A framework for automatic IT architecture modeling: applying truth discovery" takes also an architectural concern - automating IT architecture modeling. The approach is benefiting from using multiple data sources, as well as from being able to support the creation of more than one model representing multiple enterprise domains. The scope or the IT architecture modeling spans application components, data, users and IT infrastructure on a

\footnotetext{
* Corresponding author

(C) 2019 Jelena Zdravkovic. This is an open access article licensed under the Creative Commons Attribution License (http://creativecommons.org/licenses/by/4.0).

Reference: J. Zdravkovic, "Selected Topics on Business Informatics: Editorial Introduction to Issue 20 of CSIMQ," Complex Systems Informatics and Modeling Quarterly, CSIMQ, no. 20, pp. I-II, 2019. Available: https://doi.org/10.7250/csimq.201920.00

Additional information. Author's ORCID iD: J. Zdravkovic - https://orcid.org/0000-0002-0870-0330. PII S225599221900114X. Received: 30 October 2019. Available online: 31 October 2019.
} 
holistic enterprise level. Thus the framework supports inclusion of all the main layers of enterprise architecture. To ensure successful data integration, different data sets are given, a common semantic and structural representation, by transforming data to the same abstraction level and the conflicts between different data sets are resolved. In addition, because the data about various aspects of an enterprise are of varying actuality and coverage, the framework labels and weights varying degrees of trustworthiness.

"Method Framework for Developing Enterprise Architecture Security Principles" is the third article arguing that the security and privacy considerations should be embedded in all the areas of organizational activities, i.e. beyond technical security mechanisms provided by the underlying systems and software. Enterprise Architecture Management (EAM) offers a holistic approach for managing different dimensions of an organization, and can be conceived as a coherent and consistent set of principles that guide how the enterprise must be designed. The study proposes a method framework for integrating information security with Enterprise Architecture Management, aimed at providing support for the decision-making related to formulating context-aware EA security principles. The framework is reviewed by interviewing a number of Finnish EA and information security practitioners.

The fourth article "Fraud Detection in Payments Transactions: Overview of Existing Approaches and Usage for Instant Payments" takes the concern to digitalization in banking, with a particular focus on fraud discovery and the automation of that task. Fraud discovery in financial transactions has become an important priority for banks. The study proposes the use of artificial intelligence in IP fraud detection with a solution for technological support for discovering fraud detection of instant payment transactions.

The Issue 20 of CSIMQ closes with the article "Analyze \& Focus Your Intention as the First Step for Applying the Digital Innovation and Transformation Process in Zoos" which, as the previous article, focuses on digitalization. In particular, it analyzes digitalization of operational processes, continuous collection and evaluation of data about product use and evaluation of customer data or activities. The study describes the application of the digital innovation and its supporting transformation in animal-gardening facilities, as it collects, analyzes and structures information of 19 facilities within the "Landeszooverband Mecklenburg-Vorpommern e.V." and combines them with qualitative studies.

For this thematic issue we owe thanks to the members of CSIMQ's Editorial Review Board for providing valuable reviews for the submitted articles. Special thanks go to the Managing Editor of the CSIMQ - Prof. Marite Kirikova for her great support in realizing this thematic issue, as well as to the publicizing team of the CSIMQ journal for their professional help and efficiency. Finally, we are grateful to the authors for submitting the articles reporting their scientifically innovate and passionate work.

To all readers - we hope you will enjoy this issue and use it to conceive novel ideas in the actual topics of Business Informatics. 